\title{
Cryo-ET Workflow for Understanding Ion Channels Localization on the Nodes of Ranvier
}

\author{
Jiaxuan Wang \\ School of Life Science, Shandong Normal University, Jinan, China \\ Email: *wangjiaxuan190716@outlook.com
}

How to cite this paper: Wang, J.X. (2020) Cryo-ET Workflow for Understanding Ion Channels Localization on the Nodes of Ranvier. Journal of Biosciences and Medicines, 8, 55-72.

https://doi.org/10.4236/jbm.2020.82005

Received: December 20, 2019

Accepted: February 4, 2020

Published: February 7, 2020

Copyright $\odot 2020$ by author(s) and Scientific Research Publishing Inc. This work is licensed under the Creative Commons Attribution International License (CC BY 4.0).

http://creativecommons.org/licenses/by/4.0/ (c) (i) Open Access

\begin{abstract}
The localization of ion channels on myelinated axon is closely related with the saltatory conduction of action potential (AP). Abnormal changes in these channels contribute to multiple mental diseases. The development of cryo-Electron Tomography (cryo-ET) has provided a promising prospect for peering into ion channels in their native environment at high resolution. Previous achievements are reviewed here on cryo-ET. Accordingly, a cryo-ET workflow is designed for understanding ion channels localization in myelinated axon, especially nodes of Ranvier, which are significant for the saltatory conduction involved in the propagation of high-speed AP. The workflow is divided into six parts: the preparation of neural cultures with myelin, antibodies and immunofluorescence staining, frozen-hydrated sample preparation, cryo-ET imaging, cryo-correlative light and electron microscopy (cryo-CLEM) imaging, three-dimensional (3D) reconstruction and refinement. The purpose is to conceive a possible solution for the problems related to ion channel compounds including localization, conformation dynamics, accessory structures of ion channel and transient regulatory factors, and thus provide insights into treating neurological diseases caused by abnormal ion channels activity.
\end{abstract}

\section{Keywords}

Cryo-ET, Ion Channels Localization, The Nodes of Ranvier

\section{Introduction}

The normal function of the brain relies on accurate and rapid conduction of electrical signal, which is called action potential (AP). High-speed AP propagation is achieved as the saltatory conduction in myelinated axons with the regularly embedded nonmyelinated constructions, nodes of Ranvier [1] [2]. Various 
types of sodium channels and potassium channels exist in myelinated axons and cluster in nodes of Ranvier to facilitate impulse conduction by lowering space requirement as compared with the axons without myelin, and some of these channels such as rectifier $\mathrm{K}^{+}$channel help to maintain the resting potential and stabilize conduction [3]. Reasonably, subtle changes in the dynamics of these channels may give rise to faults in saltatory conduction, thus contribute to multiple psychiatric diseases [4]. To be specific, abnormality in the permeability, expression level or electrical excitability of sodium voltage-gated channels assembled with the first or sixth type of $\alpha$-subunit, namely $\mathrm{Na}_{\mathrm{v}} 1.1$ or $\mathrm{Na}_{\mathrm{v}} 1.6$ channels, at nodes of Ranvier lead to autism, epilepsy syndromes, fibromyalgia and periodic paralysis [5]; and similarly, the pathogenesis of myokymia and benign familial neonatal convulsions is closely related to mutant potassium voltage-gated channel subfamily Q member 2 (KCNQ2) and member 3 (KCNQ3) channels [6]. Also, blocking juxtaparanodal potassium voltage-gated channel subfamily A (KCNA/ $\left.\mathrm{K}_{\mathrm{v}} 1\right)$ channels by auto-antibodies contributes to limbic encephalitis and Morvan's syndrome [7], and the list can go on. Researches on the synaptic activities or localization and regulation process of ion channels on myelinated axons have provided valuable insights into the mechanism of mental disorders and potential therapeutic targets, especially with recent optimizations of data acquisition and processing in cryo-electron tomography to obtain the three-dimensional information at subnanometer resolution [8] [9] from exquisitely preserved frozen-hydrated specimens in a close-to-life state. This paper aims to review antecedent achievements, design a workflow for mapping ion channels at the nodes of Ranvier to resolve the events underlying the saltatory conduction, and determine the still missing parts of ion channel complex as the possible topics of future exploration, for reference in treating neurologic diseases either by means of drugs or neural stem cell transplantation.

\section{The Nodes of Ranvier}

The nodes of Ranvier are small about $1 \mu \mathrm{m}$ long gaps [4] along myelinated axons where there is a break in the myelin sheath and the axon membrane is in contact with the extracellular space [4], which is also called extracellular matrix (ECM) and filled with highly charged material [3] allowing $\mathrm{Na}^{+}$entry through voltage-gated channels [4]. Nodes of Ranvier are bordered by the paranodal axoglial junctions (PNJ), a specialized axon-glial contact formed between the axolemma and the paranodal loops which are formed by myelin lamellae and closely opposed to the axon with a gap of only $2.5-3 \mathrm{~nm}$ [3]. These loops spiral around the axon and form a series of ladder-like junctions with the axon.

Various types of $\mathrm{Na}^{+}$and $\mathrm{K}^{+}$channels cluster at nodes of Ranvier [3] including Nav1.1, Nav1.2, Nav1.6, Nav1.7, Nav1.8, Nav1.9 [10] [11] [12] [13] [14], and Kv3.1b, KCNQ2, KCNQ3 [15] [16]. These $\mathrm{Na}^{+}$channels also interact with the accessory $\beta$-subunits such as Navb1, Navb2, and Navb4 [17] [18] [19]. The $\beta$-subunits are covalently linked to $\mathrm{Na}^{+}$channels through an extracellular disul- 
fide bond [19] [20] to promote the surface expression of $\mathrm{Na}^{+}$channels and change their biophysical properties.

\section{The Advantages and Recent Optimization of Cryo-ET}

Cryo-Electron Tomography (cryo-ET) is a powerful technology to image frozenhydrated specimens with a transmission electron microscope (TEM) in a range of tilt angles and derive the three-dimensional (3D) information of objects in their native conditions [21]-[30]. Based on conventional TEM, cryo-ET has made improvements on sample preparation and data collection, and acquired multiple advantages in biological research. First, cryofixation circumvents most of the artifacts by stabilizing molecules in specimen instantaneously. Fine structures of the biological material can be exquisitely preserved for future scrutiny in TEM [31] [32]. Second, the sample preparation of cryo-ET does not demand isolating the object of study from its native context [23]. In the cryo-ET workflow, a specific specimen are cryofixed and imaged without rigorous purification, and thus, peering into the complete spectrum of interactions relating to the interested structure is possible [21] [23] [33]-[36]. Third, the electron tomography combined with subtomogram averaging produces $3 \mathrm{D}$ reconstruction of a unique biological specimen at subnanometer resolution [8] [9] [37]-[41].

Bracing advances in cryo-ET have already been underway, pushing the biological imaging to even state-of-the-art resolution. Focused ion beam (FIB), as a well-established technique in the semiconductor industry and material science, is emerging to be a highly reliable method for the sample preparation when jointed with cryo-ET [42]. Cryo-FIB has effectively improved the image quality by preventing artifacts from knife-cuts such as compression in the cutting direction, curved sections and crevasses [43]. The advantages of cryo-FIB have been extended by its affiliation with fluorescence microscopy, that is the correlated light and electron microscopy (CLEM), to target fluorescently-labelled proteins in cryo-FIB milled sample. Also, new software and hardware for semi-automated imaging is developed for cryo-CLEM, improving the imaging throughout and fidelity by offering high stability and contamination-free loading of sample [44]. In addition, the application of direct detection cameras in cryo-technique has remarkably reduced the total electron dose and the acquisition time for tomographic data collection, thus facilitating the ultra-low-dose imaging for beamsensitive specimens [45]. The Volta phase plate (VPP) is also incorporated into imaging workflow to largely enhance the phase contrast especially towards fine specimen structures, and the relevant workflow has been improved to include a conductive-coating procedure on sample to remove the adverse charging in lamellas for VPP imaging [46]. To maximize the high-resolution information available from tomograms for subsequent analysis, a dose-symmetric tilt-scheme is proposed that tilt-scheme starts from zero degree, and then moves up to the highest by alternating between the positive and negative tilts simultaneously [8]. In this way, additional information can be collected from the lower tilts when the radia- 
tion damage hasn't accumulated and the sample is at its thinnest state so that the information collected is of high-resolution [8].

\section{Cryo-ET Workflow of Studying Ion Channel at the Nodes of Ranvier}

The overall workflow of cryo-ET studying ion channel at the nodes of Ranvier is illustrated in Figure 1(a). Neuronal cultures are grown on EM grids and then plunge frozen for cryo-ET imaging followed by $3 \mathrm{D}$ reconstruction. For cultures transfected with DNA constructs of fluorescent protein-tagged ion channel component, cryo-fluorescence microscopy is performed before cryo-ET for correlative imaging [47].

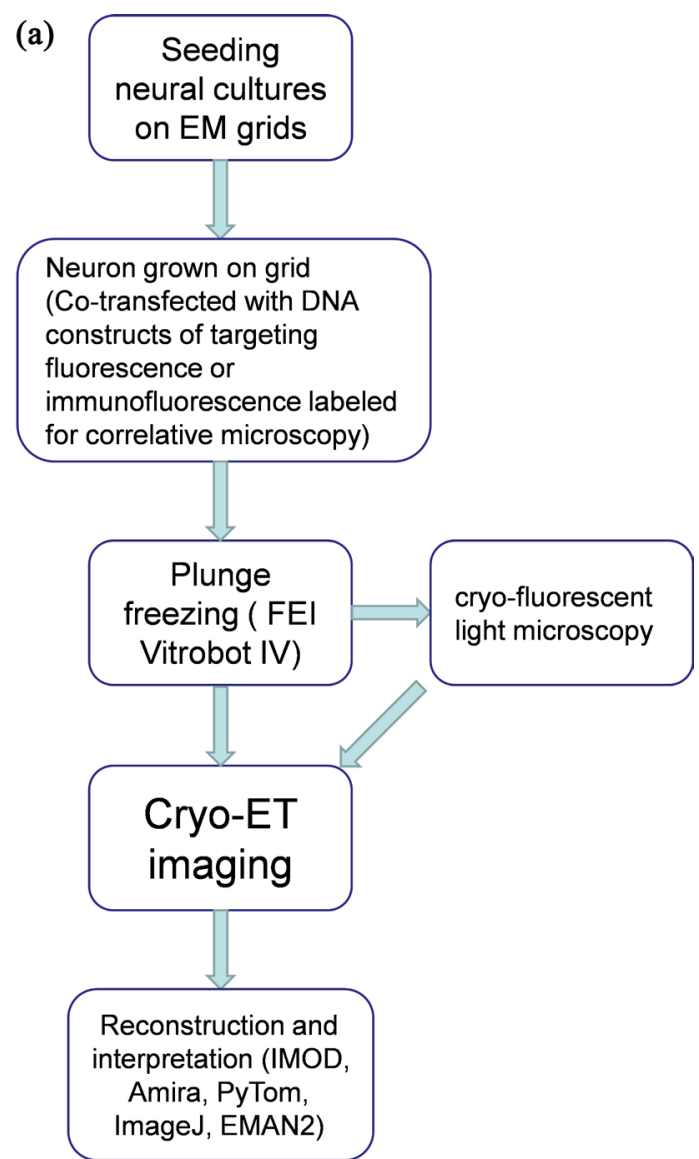

(b)

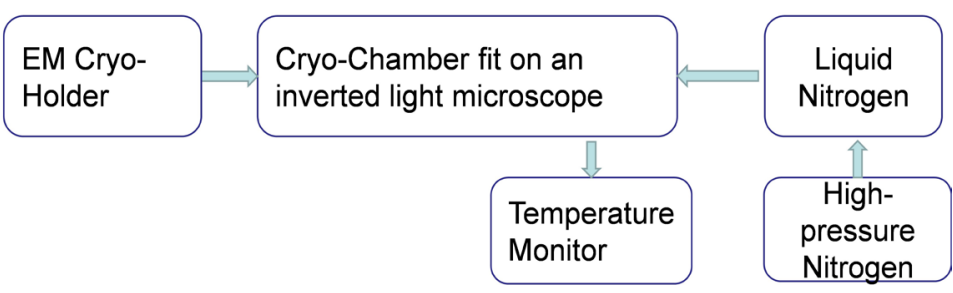

Figure 1. The cryo-ET/cryo-CLEM workflow of studying ion channel at the nodes of Ranvier. (a) Illustration of the workflow of cryo-ET/cryo-CLEM imaging of neurons grown on EM grids. (b) Schematics depicting main components of cryo-fluorescence light microscope with an EM cryo-holder. 
The preparation of neural cultures with myelin. The experimental animal absent of specific proteins can be gained through gene knockout which may be confirmed by Western blot analysis, and the sample source can be embryonic, such as dissociated primary mixed hippocampal cells [48] [49] or spinal cord cells [50] [51] for the central nervous system (CNS) study. As for the study of nodes of Ranvier in the peripheral nervous system (PNS), dissociated dorsal root ganglia myelinating cultures can be prepared as described previously [50] [52] [53]. The detached cells are seeded on poly-L-lysine coated electron microscope (EM) grids such as Quantifoil R2/2 gold EM grids or Quantifoil R2/2 gold NH2 finder grids in Petri dishes at proper density, and incubated at $37^{\circ} \mathrm{C}$ in $5 \% \mathrm{CO}_{2}$ with the culture medium replaced regularly [47]. For correlative microscopy, cultures are transfected with lentiviruses [47] containing the DNA construct of targeting fluorescence such as yellow fluorescent protein (YFP)-Kv1.2, cyan fluorescent protein (CFP)-Kv1.2, YFP-Kv $\beta 2$ or CFP-Kv $\beta 2$ DNA construct [54].

Antibodies and immunofluorescence staining. When the culture checked under phase-contrast light microscope [47] is healthy and ready for subsequent sample preparation, immunolabeling of ion channels with antibody may be applied on the culture for future CLEM study. Primary antibodies may include mouse anti- $\mathrm{Na}_{\mathrm{v}}$ pan, rabbit anti- $\mathrm{Na}_{\mathrm{v}} 1.6$ [55], mouse anti- $\mathrm{K}_{\mathrm{v}} 1.1$, anti- $\mathrm{K}_{\mathrm{v}} 1.2$, anti- $\mathrm{K}_{\mathrm{v}} 1.4$ [48] and anti- $\mathrm{K}_{\mathrm{v}} \beta 2$ [49]. Retigabine and linopirdine also bind to KCNQ channels specifically [16]. Appropriate secondary antibody such as immunoglobulin $G$ (IgG) or anti-rabbit conjugated with Alexa Fluor or cyanine dyes can be added to the culture for immunofluorescence labeling after redundant primary antibody is washed off with buffer solution [49] [56]. The various types of antibodies are clearly listed in Table 1 for reference.

Frozen-hydrated sample preparation. Low-density neuronal cultures grown on EM grids taken from the culture incubator are first placed in extracellular solution then mounted on a FEI Vitrobot IV. Protein A-coated colloidal gold beads $(10-20 \mathrm{~nm})$ are added to the grid as fiducial markers. After several seconds of back blotting with the aid of Teflon sheets [46], the grids are flash frozen in the liquid ethane or ethane/propane mixture for rapid vitrification. The samples are then stored in liquid nitrogen until use [47].

The sample may be thinned by FIB micromachining. Cryo-FIB has effectively improved the image quality by preventing artifacts from knife-cuts, and the workflow of cryo-FIB has been optimized by coating the prepared lamella with a thin layer of platinum to improve conductivity so that the subsequently reconstructed volumes obtained with VPP may exhibit high contrast. In some cases, to avoid the small contrast reduction caused by Pt coating, a sandwich-type pre-milling coating might be considered-a conventional sputtered Pt layer followed by an organometallic protective layer, and then a final Pt layer is deposited.

Cryo-ET imaging. Grids are transferred to transmission electron microscope operating at acceleration voltage of $300 \mathrm{keV}$ equipped with a direct electron detector set to a pixel size of $1.6 \AA$ - $5 \AA$ [57] [58], and for each reconstruction of 
Table 1. The primary antibody and secondary antibody available for immunolabeling of ion channels.

\begin{tabular}{|c|c|c|}
\hline Ion channel & Specific primary antibody for ion channel & Secondary antibody \\
\hline $\mathrm{Na}_{\mathrm{v}}$ & Mouse anti-Na $\mathrm{Na}_{\mathrm{v}}$ pan & \multirow{17}{*}{$\begin{array}{c}\text { IgG or anti-rabbit conjugated } \\
\text { with Alexa Fluor or cyanine } \\
\text { dyes }\end{array}$} \\
\hline $\mathrm{Na}_{\mathrm{v}} 1.1$ & Guinea pig anti- $\mathrm{Na}_{\mathrm{v}} 1.1$ & \\
\hline $\mathrm{Na}_{\mathrm{v}} 1.2$ & Guinea pig anti- $\mathrm{Na}_{\mathrm{v}} 1.2$ & \\
\hline $\mathrm{Na}_{\mathrm{v}} 1.6$ & Rabbit anti-Nav 1.6 & \\
\hline $\mathrm{Na}_{\mathrm{v}} 1.7$ & Guinea pig anti- $\mathrm{Na}_{\mathrm{v}} 1.7$ & \\
\hline $\mathrm{Na}_{\mathrm{v}} 1.8$ & Guinea pig anti- $\mathrm{Na}_{\mathrm{v}} 1.8$ & \\
\hline $\mathrm{Na}_{\mathrm{v}} 1.9$ & Guinea pig anti- $\mathrm{Na}_{\mathrm{v}} 1.9$ & \\
\hline $\mathrm{K}_{\mathrm{v}} 1.1$ & Mouse anti- $\mathrm{K}_{\mathrm{v}} 1.1 /$ Rabbit anti- $\mathrm{K}_{\mathrm{v}} 1.1$ & \\
\hline $\mathrm{K}_{\mathrm{v}} 1.2$ & Mouse anti- $\mathrm{K}_{\mathrm{v}} 1.2$ & \\
\hline $\mathrm{K}_{\mathrm{v}} 1.4$ & Mouse anti- $\mathrm{K}_{\mathrm{v}} 1.4$ & \\
\hline $\mathrm{K}_{\mathrm{v}} \beta 2$ & Mouse anti- $\mathrm{K}_{\mathrm{v}} \beta 2$ & \\
\hline KCNQ & Retigabine and linopirdine & \\
\hline KCNQ2 & Anti-KCNQ2 & \\
\hline Neurofascin & Anti-neurofascin & \\
\hline PSD-93 & Anti-PSD-93 & \\
\hline Caspr2 & Anti-Caspr2 & \\
\hline TRAAK & Anti-TRAAK & \\
\hline
\end{tabular}

tomogram, a series of pictures are taken at regular tilt increments in an automated fashion. Tilt series may be recorded with an angular increment of $2^{\circ}$ from $-60^{\circ}$ to $+60^{\circ}$, using a computer-controlled goniometer to accurately increment the angular steps [59]. To maximize the high-resolution information available from tomograms, a dose-symmetric tilt-scheme can be applied to collect additional high-resolution information from the lower tilts when the radiation damage hasn't been accumulated and the sample is at its thinnest state [8]. The images are acquired with VPP [60], providing enhanced contrast.

Cryo-correlative light and electron microscopy imaging. A specially designed CLEM system is required to include a custom-built cryo-chamber with liquid nitrogen supply, a cryo-holder, and an inverted fluorescent microscope, as illustrated in Figure 1(b) [47]. The inside channel of the cryo-chamber is precooled to $-190^{\circ} \mathrm{C}$ by liquid nitrogen, and maintained below $-180^{\circ} \mathrm{C}$, as monitored by a thermoelectric sensor. Then, an EM grid with frozen-hydrated sample is loaded onto an EM cryo-holder, which is subsequently inserted into the cryo-chamber [47]. Fluorescence images are taken both in bright field and the fluorescence channel, and areas of the sample imaged are identified in the EM using the indexes of the finder grids [47]. Low-magnification EM images are collected and approximately aligned with bright-field light microscope images using Midas program in the IMOD package [61]. Tilt series are collected on the area with se- 
lected fluorescent signals, and subsequently fine-aligned and merged with the fluorescence images to identify the segment of interest using Midas and ImageJ [47].

$3 D$ Reconstruction and Refinement. The overall workflow of image processing and related softwares are presented in Figure 2. Tilt series are aligned and reconstructed into 3D tomograms using IMOD [61]. The gold beads added to the sample before plunge freezing are used as fiducial markers to align the tilt series. Tilt image alignment includes refinement of tilt axis angle and tilt angles, determination of image shifts, and accounting for beam-induced sample deformation [8]. MotionCor2 is robust, and sufficiently accurate at correcting local motions [62]. Images are CTF-corrected by phase-flipping before reconstruction of the 3D volumes [63] and the parameters of the CTF are estimated by ctffind4 [64]. Reconstruction is performed using a simultaneous iterative reconstruction technique of IMOD with 5 or 15 iterations [47].

Segmentation can be defined as a process by which electron-dense features in a tomogram are ascribed identities and highlighted relative to other features [65]. Automatic segmentation of specific features is realized by EMAN2.2 software package based on convolutional neural networks to dramatically reduce the time and human effort required for subcellular annotation and feature extraction [66]. Nonetheless, tomograms are often segmented manually because the available segmentation algorithms are often inferior to human anticipation, which can infer

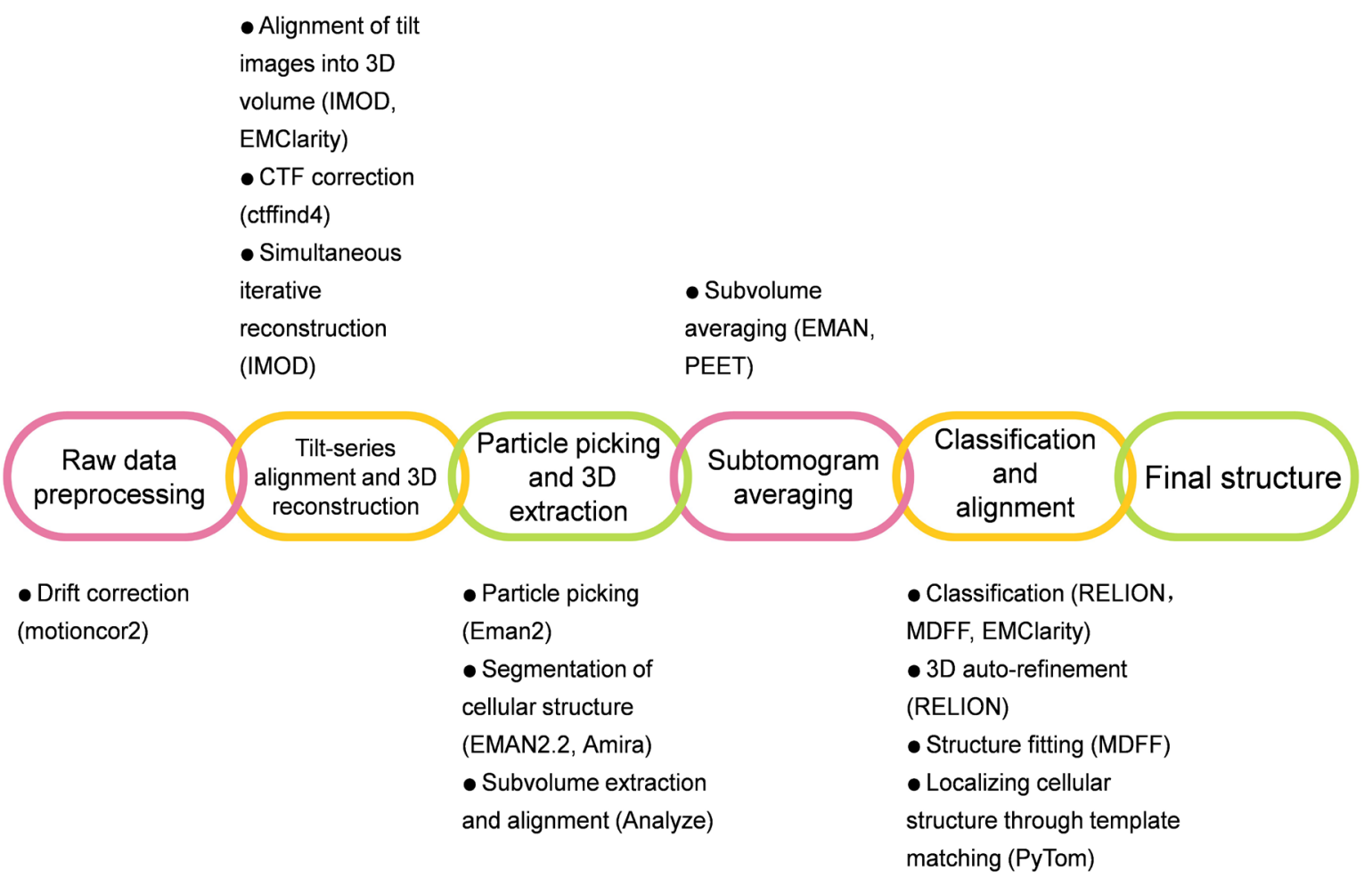

Figure 2. The overall workflow of image processing and pertinent softwares. The image processing mainly includes raw data preprocessing, tilt-series alignment and $3 \mathrm{D}$ reconstruction, particle picking and $3 \mathrm{D}$ extraction, subtomomgram averaging, classification and alignment. 
incomplete or anisotropic data [65]. Volume-rendered segmentations can also be manually performed using the Amira package [67]. The volume of each structure is displayed according to the intensity value [47] and filtered to make the densities smooth and continuous. Visualization and movie making can be carried out in IMOD and Amira software packages [63]. The measurement of structural features can be achieved with ImageJ [59].

Subsequent subtomogram averaging and classification yield in situ structures of molecular components of interest. A tomogram of a cell contains densities corresponding to the proteome. If a template structure generated by an independent technique is modified to suit the characteristics of the tomogram, then the tomogram can be searched exhaustively using this structure as a template. Templates can be obtained from the tomogram too [65]. A requirement of subtomogram averaging is that the features recognizable in the tomograms [65]. After localizing the repetitive features manually or via template matching, subvolumes of similar dimension are cut out and aligned together in 3D with Analyze [59], and multiple subvolumes are averaged afterwards using EMAN [65] [68] [69] or PEET [70] to increase the signal-to-noise ratio, enabling visualization of greater details [59].

The high-resolution features such as the structures of protein subunits can be classified including $2 \mathrm{D}$ classification of projections and 3D classification, and 3D auto-refined by RELION [71] [72]. For each class, molecular dynamics flexible fitting (MDFF) is performed to refine functional models according to the density maps [73]. Also, the physiological role of different protein conformations may be addressed by mapping the particles back to the tomograms to investigate their cellular distribution [73]. Particles of interest such as ion channels from a subset of the images can be auto-selected using EMAN2 to generate templates representing different views for automated particle selection [69] [74] and manually inspected to remove false positives afterwards [58]. The template can be used to localize identical structures in sample with PyTom [75]. EMClarity is also developed to enhance macromolecular classification and alignment for high-resolution tomography [76]. The segmented volume of ion channel can be fitted into protein data bank to define new, unknown density that is extra to the existing ion channel. Then the specific protein complex may be isolated from cell membrane and analyzed with Mass spectrometry to identify new interactions among the components of protein complex such as temporary regulatory proteins or cell specific protein subunits.

\section{The Application of Similar Workflow in Neuroscience}

In the field of neuroscience, similar devised workflow of cryo-ET has been applied on studying the ultrastructure of myelin, synapses and synaptic activity such as neurotransmitter release, as well as some protein aggregates involved in neuropathy.

When it comes to exploring the genesis and structure of myelin, cryo-ET defi- 
nitely plays a crucial role by maintaining state-of-the-art sample and imaging of axon and myelin unit. For example, the filamentous cross-bridges connecting cytoskeleton or membranes in the paranodes of the PNS have been ultrastructurally characterized in 3D with cryo-ET. These filaments form connections between the cytoskeleton of paranodal loop and that in the axon, and tether cytoplasmic organelles as well as other membranous organelles to cytoskeleton or membrane [59]. Besides, some marker such as myelin basic protein (MBP) can be identified and tracked for dynamic development of myelin at the ultrastructural level, and the cryo-ET study of MBP has provided new evidence for the developmental model that the formation of the myelin major dense line during myelination is driven by MBPs [57].

The neural structures involved in presynaptic and cytoskeletal organization, axonal transport and endocytosis can be quantitatively characterized in a closeto-native state with cryo-ET. For instance, the structure, organization and localization of microtubules, vesicles and smooth endoplasmic reticulum in presynapses may be readily identifiable in 3D segmentation of complete axonal boutons from tomographic slices. It has been found that short pleomorphic linkers extensively interconnect vesicles and lipid membranes, and may serve as organizers among these components in neurons [56]. Cryo-ET has been applied in studying calcium-regulated exocytosis taking place between $200 \mu \mathrm{s}$ and $1 \mathrm{~ms}$ [77]. The organization of the release machinery under the docked synaptic vesicles (SVs) is delineated in nerve growth factor-differentiated neuro-endocrine (PC12) cells, and it has been observed that six distinct modules are arranged symmetrically at the SV-plasma membrane (PM) interface [77]. Vesicle-associated membrane protein 2 (VAMP2) in SVs are fluorescence labeled for CLEM study by transfecting PC12 with VAMP2-4X-pHluorin constructs [77]. The C-terminal region of Munc13-1 protein encompassing the $\mathrm{C} 1, \mathrm{C} 2 \mathrm{~B}, \mathrm{MUN}$ and $\mathrm{C} 2 \mathrm{C}$ domains is proposed to bridge $\mathrm{SV}$ and $\mathrm{PM}$ through interactions involving the $\mathrm{C} 2 \mathrm{C}$ domain and the $\mathrm{C1}-\mathrm{C} 2 \mathrm{~B}$ region [78]. This model is supported by the cryo-ET images which provide a direct visualization of how the Munc13-1 C1C2BMUNC2C fragment can bridge two membranes through sequences located at opposite ends of the MUN domain based on dynamic light scattering experiments [78] [79].

Cryo-ET can also be exerted on the study of protein aggregates which might be implicated in some neuropathy. For example, the poly-Gly-Ala (poly-GA) aggregates are associated with various kinds of neural diseases such as frontotemporal dementia and amyotrophic lateral sclerosis. The structural study with cryo-ET indicates that compact twisted ribbons constitute poly-GA aggregates, and the impairing impact on proteasomes has been deduced from the particular structure of these aggregates [73].

In brief, it's remarkably promising to apply cryo-ET to study biological structure in great detail and of high fidelity considering the ongoing advances in cryotechnique and antecedent achievements. In the field of neuroscience, cryo-ET has a even greater role to play to dig into the complicated interactions among 
neural components such as those factors involved in the deteriorating and developmental process of myelin, which is of great significance for understanding the mechanism of demyelinating diseases and inventing effective treating strategies.

\section{Possible Directions in Studying the Structure and Conformation Dynamics of Ion Channel Assembly on Myelinated Axons in Terms of Using Cryo-ET as an Approach}

Proteomics of Kv1 channels revealed ADAM22 at juxtaparanodes (JXP) [80]. However, it remains unknown how ADAM22 is recruited, what are the function and ligands of ADAM22 as well as how Kv1 channels interact with cell adhesion molecules (CAMs) such as Caspr2 and TAG-1 [3]. The PDZ-domain proteins PSD95 and PSD93 also exist in Kv1 channel complex with the function of PSD95/93 remains to be studied [3]. Three types of CAMs, $155-\mathrm{kDa}$ isoform of neurofascin (NF155), Caspr and contactin [81], mediate axon-glia interaction in the PNJ, separate JXP proteins from nodes of Ranvier [3], and thus contribute to the maintenance of ion channel distribution. And yet, the cytoplasmic partners of NF155 are currently unknown [3]. The other CAMs such as neuron-glia related cell adhesion molecules (NrCAMs) and the $186-\mathrm{kDa}$ isoform of neurofascin (NF186) also play a significant role in linking scaffolds of ion channel to extrinsic interactions at nodes of Ranvier [82], although in the CNS, the cellular source of shed NrCAMs in the nodal ECM is still unknown [3]. With the help of cryo-ET, the auxiliary structures of ion channel in terms of their source, corresponding ligands and function can be studied in native environment. The relevant research is important for understanding how ion channels are assembled and maintained, and provides insights into how these micromachines can be repaired after injury [3].

The identification of side-chains and the structural determination of amphipols-stabilized membrane proteins have already been achieved by cryo-EM especially when the use of detergents is undesirable [83]. With the developments ushering in the resolution revolution including the use of direct electron detector, VPP and focused ion beam milling, cryo-ET is extremely beneficial for mapping the localization of ion channel on the myelinated axon as well as investigating the conformation dynamics of native ion channels such as the transient curvature of lipid bilayer, arrangement of transmembrane helix, and the folding state of inactivated or desensitized ion channels. Cryo-ET may solve the problem of conformation change by dissecting the channel gating motions on the millisecond timescale [83].

Myelination can be modulated by extrinsic factors including electrical activity of axons, acting via oligodendroglial calcium transients [84] [85], the glutamatergic stimulation of glucose uptake [86] and other extracellular signalling molecules [87] [88]. Studying ion channel localization on myelinating axon and signaling pathway for growing myelin can be achieved well with cryo-ET in terms of imaging the objects in native environment and the preservation of transient 
signaling factors. Further insights into the mechanism of myelinating process will be instrumental for treatment of cerebropathy during neural development and restoration of adult myelin.

\section{Conclusion}

In this paper, antecedent works on the merits and applications of cryo-ET in neurobiology are reviewed, aiming to design a practical workflow for studying ion channels at nodes of Ranvier. Multiple improvements in cryo-ET are emphasized such as cryo-FIB, cryo-CLEM, and dose-symmetric tilt-scheme method for data collecting, which are significant in broadening the application of cryo-ET and enhancing the resolution of output. Overall, cryo-ET is a promising technique for solving the problems related to ion channel compounds including localization, conformation dynamics, accessory structures of ion channel and transient regulatory factors, due to the advantages of cryo-ET by allowing studies of biological ultrastructure in its native conditions and the collection of high-resolution 3D information of objects.

\section{Conflicts of Interest}

The author declares no conflicts of interest regarding the publication of this paper.

\section{References}

[1] Ranvier, L. (1871) Contributions à l'histologie et à la physiologie des nerfs périphériques. Comptes Rendus de 1 Académie des Sciences, 73, 1168-1171.

[2] Ranvier, L. (1872) Recherches sur l'histologie et la physiologie des nerfs. Archive de physiologie normale et pathologique, 4, 129-149.

[3] Rasband, M.N. and Peles, E. (2015) The Nodes of Ranvier: Molecular Assembly and Maintenance. Cold Spring Harbor Perspectives in Biology, 8, a020495. https://doi.org/10.1101/cshperspect.a020495

[4] Arancibia-Carcamo, I.L. and Attwell, D. (2014) The Node of Ranvier in CNS Pathology. Acta Neuropathologica, 128, 161-175.

https://doi.org/10.1007/s00401-014-1305-Z

[5] Eijkelkamp, N., Linley, J.E., Baker, M.D., Minett, M.S., Cregg, R., Werdehausen, R., Rugiero, F. and Wood, J.N. (2012) Neurological Perspectives on Voltage-Gated Sodium Channels. Brain, 135, 2585-2612. https://doi.org/10.1093/brain/aws225

[6] Maljevic, S., Wuttke, T.V. and Lerche, H. (2008) Nervous System KV7 Disorders: Breakdown of a Subthreshold Brake. The Journal of Physiology, 586, 1791-1801. https://doi.org/10.1113/jphysiol.2008.150656

[7] Kleopa, K.A., Elman, L.B., Lang, B., Vincent, A. and Scherer, S.S. (2006) Neuromyotonia and Limbic Encephalitis Sera Target Mature Shaker-Type $\mathrm{K}^{+}$Channels: Subunit Specificity Correlates with Clinical Manifestations. Brain, 129, 1570-1584. https://doi.org/10.1093/brain/awl084

[8] Hagen, W.J.H., Wan, W. and Briggs, J.G. (2017) Implementation of a Cryo-Electron Tomography Tilt-Scheme Optimized for High Resolution Subtomogram Averaging. Journal of Structural Biology, 197, 191-198.

https://doi.org/10.1016/j.jsb.2016.06.007 
[9] Pfeffer, S., Burbaum, L., Unverdorben, P., Pech, M., Chen, Y., Zimmermann, R., Beckmann, R. and Forster, F. (2015) Structure of the Native Sec61 Protein-Conducting Channel. Nature Communications, 6, 8403. https://doi.org/10.1038/ncomms9403

[10] Black, J.A., Frezel, N., Dib-Hajj, S.D. and Waxman, S.G. (2012) Expression of Nav1.7 in DRG Neurons Extends from Peripheral Terminals in the Skin to Central Preterminal Branches and Terminals in the Dorsal Horn. Molecular Pain, 8, 82. https://doi.org/10.1186/1744-8069-8-82

[11] Boiko, T., Rasband, M.N., Levinson, S.R., Caldwell, J.H., Mandel, G., Trimmer, J.S. and Matthews, G. (2001) Compact Myelin Dictates the Differential Targeting of Two Sodium Channel Isoforms in the Same Axon. Neuron, 30, 91-104. https://doi.org/10.1016/S0896-6273(01)00265-3

[12] Duflocq, A., Le Bras, B., Bullier, E., Couraud, F. and Davenne, M. (2008) Nav1.1 Is Predominantly Expressed in Nodes of Ranvier and Axon Initial Segments. Molecular and Cellular Neuroscience, 39, 180-192. https://doi.org/10.1016/j.mcn.2008.06.008

[13] Fjell, J., Hjelmstrom, P., Hormuzdiar, W., Milenkovic, M., Aglieco, F., Tyrrell, L., Dib-Hajj, S., Waxman, S.G. and Black, J.A. (2000) Localization of the Tetrodotoxin-Resistant Sodium Channel NaN in Nociceptors. NeuroReport, 11, 199-202. https://doi.org/10.1097/00001756-200001170-00039

[14] Henry, M.A., Sorensen, H.J., Johnson, L.R. and Levinson, S.R. (2005) Localization of the Nav1.8 Sodium Channel Isoform at Nodes of Ranvier in Normal Human Radicular Tooth Pulp. Neuroscience Letters, 380, 32-36. https://doi.org/10.1016/j.neulet.2005.01.017

[15] Devaux, J., Alcaraz, G., Grinspan, J., Bennett, V., Joho, R., Crest, M. and Scherer, S.S. (2003) Kv3.1b Is a Novel Component of CNS Nodes. Journal of Neuroscience, 23, 4509-4518. https://doi.org/10.1523/JNEUROSCI.23-11-04509.2003

[16] Devaux, J.J., Kleopa, K.A., Cooper, E.C. and Scherer, S.S. (2004) KCNQ2 Is a Nodal $\mathrm{K}^{+}$Channel. Journal of Neuroscience, 24, 1236. https://doi.org/10.1523/JNEUROSCI.4512-03.2004

[17] Chen, C., Bharucha, V., Chen, Y., Westenbroek, R.E., Brown, A., Malhotra, J.D., Jones, D., Avery, C., Gillespie, P.J., Kazen-Gillespie, K.A., Kazarinova-Noyes, K., Shrager, P., Saunders, T.L., Macdonald, R.L., Ransom, B.R., Scheuer, T., Catterall, W.A. and Isom, L.L. (2002) Reduced Sodium Channel Density, Altered Voltage Dependence of Inactivation and Increased Susceptibility to Seizures in Mice Lacking Sodium Channel $\beta 2$-Subunits. Proceedings of the National Academy of Sciences of the United States of America, 99, 17072-17077.

https://doi.org/10.1073/pnas.212638099

[18] Chen, C., Westenbroek, R.E., Xu, X., Edwards, C.A., Sorenson, D.R., Chen, Y., Mcewen, D.P., O'malley, H.A., Bharucha, V., Meadows, L.S., Knudsen, G.A., Vilaythong, A., Noebels, J.L., Saunders, T.L., Scheuer, T., Shrager, P., Catterall, W.A. and Isom, L.L. (2004) Mice Lacking Sodium Channel $\beta 1$ Subunits Display Defects in Neuronal Excitability, Sodium Channel Expression and Nodal Architecture. Journal of Neuroscience, 24, 4030-4042. https://doi.org/10.1523/JNEUROSCI.4139-03.2004

[19] Buffington, S.A. and Rasband, M.N. (2013) $\mathrm{Na}^{+}$Channel-Dependent Recruitment of $\mathrm{Nav} \beta 4$ to Axon Initial Segments and Nodes of Ranvier. Journal of Neuroscience, 33, 6191-6202. https://doi.org/10.1523/JNEUROSCI.4051-12.2013

[20] Chen, C., Calhoun, J.D., Zhang, Y., Lopez-Santiago, L., Zhou, N., Davis, T.H., Salzer, J.L. and Isom, L.L. (2012) Identification of the Cysteine Residue Responsible for Disulfide Linkage of $\mathrm{Na}^{+}$Channel Alpha and $\beta 2$ Subunits. The Journal of Biological Chemistry, 287, 39061-39069. https://doi.org/10.1074/jbc.M112.397646 
[21] Lucic, V., Rigort, A. and Baumeister, W. (2013) Cryo-Electron Tomography: The Challenge of Doing Structural Biology in Situ. Journal of Cell Biology, 202, 407-419. https://doi.org/10.1083/jcb.201304193

[22] Oikonomou, C.M. and Jensen, G.J. (2017) Cellular Electron Cryotomography: Toward Structural Biology in Situ. Annual Review of Biochemistry, 86, 873-896. https://doi.org/10.1146/annurev-biochem-061516-044741

[23] Dunstone, M.A. and De Marco, A. (2017) Cryo-Electron Tomography: An Ideal Method to Study Membrane-Associated Proteins. Philosophical Transactions of the Royal Society B: Biological Sciences, 372, Article ID: 20160210. https://doi.org/10.1098/rstb.2016.0210

[24] Lucic, V., Leis, A. and Baumeister, W. (2008) Cryo-Electron Tomography of Cells: Connecting Structure and Function. Histochemistry and Cell Biology, 130, 185-196. https://doi.org/10.1007/s00418-008-0459-y

[25] Kaledhonkar, S., Fu, Z., Caban, K., Li, W., Chen, B., Sun, M. and Gonzalez, R.L. (2019) Late Steps in Bacterial Translation Initiation Visualized Using Time-Resolved Cryo-EM. Nature, 570, 400-404.

[26] Fu, Z., Indrisiunaite, G., Kaledhonkar, S., Shah, B., Sun, M., Chen, B., et al. (2019) The Structural Basis for Release-Factor Activation during Translation Termination Revealed by Time-Resolved Cryogenic Electron Microscopy. Nature Communications, 10, Article Number: 2579. https://doi.org/10.1038/s41467-019-10608-Z

[27] Qiu, W., Fu, Z., Xu, G.G., Grassucci, R.A., Zhang, Y., Frank, J., Hendrickson, W.A. and Guo, Y. (2018) Structure and Activity of Lipid Bilayer within a Membrane-Protein Transporter. Proceedings of the National Academy of Sciences, 115, 12985-12990. https://doi.org/10.1073/pnas.1812526115

[28] Kaledhonkar, S., Fu, Z., White, H. and Frank, J. (2018) Time-Resolved Cryo-Electron Microscopy Using a Microfluidic Chip. Protein Complex Assembly, 1764, 59-71. https://doi.org/10.1007/978-1-4939-7759-8 4

[29] Feng, X., Fu, Z., Kaledhonkar, S., Jia, Y., Shah, B., Jin, A., Liu, Z., Sun, M., Chen, B., et al. (2017) A Fast and Effective Microfluidic Spraying-Plunging Method for High-Resolution Single-Particle cryo-EM. Structure, 25, 663-670. https://doi.org/10.1016/j.str.2017.02.005

[30] Fu, Z., Kaledhonkar, S., Borg, A., Sun, M., Chen, B., Grassucci, R.A., Ehrenberg, M., et al. (2016) Key Intermediates in Ribosome Recycling Visualized by Time-Resolved Cryoelectron Microscopy. Structure, 24, 2092-2101. https://doi.org/10.1016/j.str.2016.09.014

[31] Villa, E., Schaffer, M., Plitzko, J.M. and Baumeister, W. (2013) Opening Windows into the Cell: Focused-Ion-Beam Milling for Cryo-Electron Tomography. Current Opinion in Structural Biology, 23, 771-777.

https://doi.org/10.1016/j.sbi.2013.08.006

[32] Wagner, J., Schaffer, M. and Fernández-Busnadiego, R. (2017) Cryo-Electron Tomography: The Cell Biology that Came in from the Cold. FEBS Letters, 591, 2520-2533. https://doi.org/10.1002/1873-3468.12757

[33] Briggs, J.A. (2013) Structural Biology in Situ-The Potential of Subtomogram Averaging. Current Opinion in Structural Biology, 23, 261-267. https://doi.org/10.1016/j.sbi.2013.02.003

[34] Leis, A., Rockel, B., Andrees, L. and Baumeister, W. (2009) Visualizing Cells at the Nanoscale. Trends in Biochemical Sciences, 34, 60-70. https://doi.org/10.1016/j.tibs.2008.10.011

[35] Wan, W. and Briggs, J.G. (2016). Chapter Thirteen-Cryo-Electron Tomography and 
Subtomogram Averaging. In: Crowther, R.A., Ed., Methods in Enzymology, Academic Press, New York, 329-367. https://doi.org/10.1016/bs.mie.2016.04.014

[36] Yahav, T., Maimon, T., Grossman, E., Dahan, I. and Medalia, O. (2011) Cryo-Electron Tomography: Gaining Insight into Cellular Processes by Structural Approaches. Current Opinion in Structural Biology, 21, 670-677. https://doi.org/10.1016/j.sbi.2011.07.004

[37] Xu, F., Fu, Z., Dass, S., Kotarba, A.M.E., Davis, J., Smith, S.O. and Van Nostrand W.E. (2016) Cerebral Vascular Amyloid Seeds Drive Amyloid $\beta$-Protein Fibril Assembly with a Distinct Anti-Parallel Structure. Nature communications, 7, 1-10. https://doi.org/10.1038/ncomms13527

[38] Fu, Z., Aucoin, D., Davis, J., Van Nostrand, W.E. and Smith, S.O. (2015) Mechanism of Nucleated Conformational Conversion of A $\beta 42$. Biochemistry, 54, 4197-4207. https://doi.org/10.1021/acs.biochem.5b00467

[39] Fu, Z., Aucoin, D., Ahmed, M., Ziliox, M., Van Nostrand, W.E. and Smith, S.O. (2014) Capping of A $\beta 42$ Oligomers by Small Molecule Inhibitors. Biochemistry, 53, 7893-7903. https://doi.org/10.1021/bi500910b

[40] Xu, F., Kotarba, A.M.E., Ou-Yang, M.H., Fu, Z., Davis, J., Smith, S.O., et al. (2014) Early-Onset Formation of Parenchymal Plaque Amyloid Abrogates Cerebral Microvascular Amyloid Accumulation In Transgenic Mice. Journal of Biological Chemistry, 289, 17895-17908. https://doi.org/10.1074/jbc.M113.536565

[41] Tang, T.C., Hu, Y., Kienlen-Campard, P., El Haylani, L., Decock, M., Van Hees, J., et al. (2014) Conformational Changes Induced by the A21G Flemish Mutation in the Amyloid Precursor Protein Lead to Increased A $\beta$ Production. Structure, 22, 387-396. https://doi.org/10.1016/j.str.2013.12.012

[42] Narayan, K. and Subramaniam, S. (2015) Focused Ion Beams in Biology. Nature Methods, 12, 1021-1031. https://doi.org/10.1038/nmeth.3623

[43] Hashemian, B., Millan, D. and Arroyo, M. (2016) Charting Molecular Free-Energy Landscapes with an Atlas of Collective Variables. The Journal of Chemical Physics, 145, Article ID: 174109. https://doi.org/10.1063/1.4966262

[44] Schorb, M., Gaechter, L., Avinoam, O., Sieckmann, F., Clarke, M., Bebeacua, C., Bykov, Y.S., Sonnen, A.F., Lihl, R. and Briggs, J.G. (2017) New Hardware and Workflows for Semi-Automated Correlative Cryo-Fluorescence and Cryo-Electron Microscopy/Tomography. Journal of Structural Biology, 197, 83-93. https://doi.org/10.1016/j.jsb.2016.06.020

[45] Migunov, V., Ryll, H., Zhuge, X., Simson, M., Struder, L., Batenburg, K.J., Houben, L. and Dunin-Borkowski, R.E. (2015) Rapid Low Dose Electron Tomography Using a Direct Electron Detection Camera. Scientific Reports, 5, 14516. https://doi.org/10.1038/srep14516

[46] Schaffer, M., Mahamid, J., Engel, B.D., Laugks, T., Baumeister, W. and Plitzko, J.M. (2017) Optimized Cryo-Focused Ion Beam Sample Preparation Aimed at in Situ Structural Studies of Membrane Proteins. Journal of Structural Biology, 197, 73-82. https://doi.org/10.1016/j.jsb.2016.07.010

[47] Tao, C.L., Liu, Y.T., Sun, R., Zhang, B., Qi, L., Shivakoti, S., Tian, C.L., Zhang, P., Lau, P.M., Zhou, Z.H. and Bi, G.Q. (2018) Differentiation and Characterization of Excitatory and Inhibitory Synapses by Cryo-Electron Tomography and Correlative Microscopy. Journal of Neuroscience, 38, 1493-1510. https://doi.org/10.1523/JNEUROSCI.1548-17.2017

[48] Bonetto, G., Hivert, B., Goutebroze, L., Karagogeos, D., Crepel, V. and Faivre-Sarrailh, C. (2019) Selective Axonal Expression of the Kv1 Channel Complex in Pre-Myelinated 
GABAergic Hippocampal Neurons. Frontiers in Cellular Neuroscience, 13, 222. https://doi.org/10.3389/fncel.2019.00222

[49] Gu, C. and Gu, Y. (2011) Clustering and Activity Tuning of Kv1 Channels in Myelinated Hippocampal Axons. The Journal of Biological Chemistry, 286, 25835-25847. https://doi.org/10.1074/jbc.M111.219113

[50] Bang, M.L., Vainshtein, A., Yang, H.J., Eshed-Eisenbach, Y., Devaux, J., Werner, H.B. and Peles, E. (2018) Glial M6B Stabilizes the Axonal Membrane at Peripheral Nodes of Ranvier. Glia, 66, 801-812. https://doi.org/10.1002/glia.23285

[51] Thomson, C.E., Hunter, A.M., Griffiths, I.R., Edgar, J.M. and Mcculloch, M.C. (2006) Murine Spinal Cord Explants: A Model for Evaluating Axonal Growth and Myelination in Vitro. Journal of Neuroscience Research, 84, 1703-1715. https://doi.org/10.1002/jnr.21084

[52] Eshed, Y., Feinberg, K., Poliak, S., Sabanay, H., Sarig-Nadir, O., Spiegel, I., Bermingham Jr., J.R. and Peles, E. (2005) Gliomedin Mediates Schwann Cell-Axon Interaction and the Molecular Assembly of the Nodes of Ranvier. Neuron, 47, 215-229. https://doi.org/10.1016/j.neuron.2005.06.026

[53] Feinberg, K., Eshed-Eisenbach, Y., Frechter, S., Amor, V., Salomon, D., Sabanay, H., Dupree, J.L., Grumet, M., Brophy, P.J., Shrager, P. and Peles, E. (2010) A Glial Signal Consisting of Gliomedin and NrCAM Clusters Axonal $\mathrm{Na}^{+}$Channels during the Formation of Nodes of Ranvier. Neuron, 65, 490-502. https://doi.org/10.1016/j.neuron.2010.02.004

[54] Gu, C., Zhou, W., Puthenveedu, M.A., Xu, M., Jan, Y.N. and Jan, L.Y. (2006) The Microtubule plus-End Tracking Protein EB1 Is Required for Kv1 Voltage-Gated $\mathrm{K}^{+}$ Channel Axonal Targeting. Neuron, 52, 803-816. https://doi.org/10.1016/j.neuron.2006.10.022

[55] Colombelli, C., Palmisano, M., Eshed-Eisenbach, Y., Zambroni, D., Pavoni, E., Ferri, C., Saccucci, S., Nicole, S., Soininen, R., Mckee, K.K., Yurchenco, P.D., Peles, E., Wrabetz, L. and Feltri, M.L. (2015) Perlecan Is Recruited by Dystroglycan to Nodes of Ranvier and Binds the Clustering Molecule Gliomedin. Journal of Cell Biology, 208, 313-329. https://doi.org/10.1083/jcb.201403111

[56] Schrod, N., Vanhecke, D., Laugks, U., Stein, V., Fukuda, Y., Schaffer, M., Baumeister, W. and Lucic, V. (2018) Pleomorphic Linkers as Ubiquitous Structural Organizers of Vesicles in Axons. PLoS ONE, 13, e0197886.

https://doi.org/10.1371/journal.pone.0197886

[57] Raasakka, A., Ruskamo, S., Kowal, J., Barker, R., Baumann, A., Martel, A., Tuusa, J., Myllykoski, M., Burck, J., Ulrich, A.S., Stahlberg, H. and Kursula, P. (2017) Membrane Association Landscape of Myelin Basic Protein Portrays Formation of the Myelin Major Dense Line. Scientific Reports, 7, 4974. https://doi.org/10.1038/s41598-017-05364-3

[58] Tao, X., Hite, R.K. and Mackinnon, R. (2017) Cryo-EM Structure of the Open High-Conductance $\mathrm{Ca}^{2+}$-Activated $\mathrm{K}^{+}$Channel. Nature, 541, 46-51. https://doi.org/10.1038/nature20608

[59] Perkins, G.A., Sosinsky, G.E., Ghassemzadeh, S., Perez, A., Jones, Y. and Ellisman, M.H. (2008) Electron Tomographic Analysis of Cytoskeletal Cross-Bridges in the Paranodal Region of the Node of Ranvier in Peripheral Nerves. Journal of Structural Biology, 161, 469-480. https://doi.org/10.1016/j.jsb.2007.10.005

[60] Danev, R., Buijsse, B., Khoshouei, M., Plitzko, J.M. and Baumeister, W. (2014) Volta Potential Phase Plate for in-Focus Phase Contrast Transmission Electron Microscopy. Proceedings of the National Academy of Sciences of the United States of America, 111, 15635-15640. https://doi.org/10.1073/pnas.1418377111 
[61] Kremer, J.R., Mastronarde, D.N. and Mcintosh, J.R. (1996) Computer Visualization of Three-Dimensional Image Data Using IMOD. Journal of Structural Biology, 116, 71-76. https://doi.org/10.1006/jsbi.1996.0013

[62] Zheng, S.Q., Palovcak, E., Armache, J.P., Verba, K.A., Cheng, Y. and Agard, D.A. (2017) MotionCor2: Anisotropic Correction of Beam-Induced Motion for Improved Cryo-Electron Microscopy. Nature Methods, 14, 331-332. https://doi.org/10.1038/nmeth.4193

[63] Mittendorf, K.F., Marinko, J.T., Hampton, C.M., Ke, Z., Hadziselimovic, A., Schlebach, J.P., Law, C.L., Li, J., Wright, E.R., Sanders, C.R. and Ohi, M.D. (2017) Peripheral Myelin Protein 22 Alters Membrane Architecture. Science Advances, 3, e1700220. https://doi.org/10.1126/sciadv.1700220

[64] Rohou, A. and Grigorieff, N. (2015) CTFFIND4: Fast and Accurate Defocus Estimation from Electron Micrographs. Journal of Structural Biology, 192, 216-221. https://doi.org/10.1016/j.jsb.2015.08.008

[65] Leis, A. (2018) Electron Tomography: A Primer. In: Hanssen, E., Ed., Cellular Imaging. Electron Tomography and Related Techniques, Springer, Switzerland, 1-32. https://doi.org/10.1007/978-3-319-68997-5 1

[66] Chen, M., Dai, W., Sun, Y., Jonasch, D., He, C., Schmid, M., Chiu, W. and Ludtke, S. (2017) Convolutional Neural Networks for Automated Annotation of Cellular Cryo-Electron Tomograms. Nature Methods, 14, 983-985. https://doi.org/10.1038/nmeth.4405

[67] Hampton, C.M., Strauss, J.D., Ke, Z., Dillard, R.S., Hammonds, J.E., Alonas, E., Desai, T.M., Marin, M., Storms, R.E., Leon, F., Melikyan, G.B., Santangelo, P., Spearman, P.W. and Wright, E.R. (2017) Correlated Fluorescence Microscopy and Cryo-Electron Tomography of Virus-Infected or Transfected Mammalian Cells. Nature Protocols, 12, 150-167. https://doi.org/10.1038/nprot.2016.168

[68] Schmid, M.F. and Booth, C.R. (2008) Methods for Aligning and for Averaging 3D Volumes with Missing Data. Journal of Structural Biology, 161, 243-248. https://doi.org/10.1016/j.jsb.2007.09.018

[69] Tang, G., Peng, L., Baldwin, P.R., Mann, D.S., Jiang, W., Rees, I. and Ludtke, S.J. (2007) EMAN2: An Extensible Image Processing Suite for Electron Microscopy. Journal of Structural Biology, 157, 38-46. https://doi.org/10.1016/j.jsb.2006.05.009

[70] Nicastro, D., Schwartz, C., Pierson, J., Gaudette, R., Porter, M.E. and Mcintosh, J.R. (2006) The Molecular Architecture of Axonemes Revealed by Cryoelectron Tomography. Science, 313, 944-948. https://doi.org/10.1126/science.1128618

[71] Bharat, T.M. and Scheres, S.H.W. (2016) Resolving Macromolecular Structures from Electron Cryo-Tomography Data Using Subtomogram Averaging in RELION. Nature Protocols, 11, 2054-2065. https://doi.org/10.1038/nprot.2016.124

[72] Martinez-Sanchez, A., Kochovski, Z., Laugks, U., Borgloh, J.M.Z.A., Pfeffer, S., Baumeister, W. and Lucic, V. (2018) Template-Free Detection and Classification of Heterogeneous Membrane-Bound Complexes in Cryo-Electron Tomograms. BioRxiv. 413484. https://doi.org/10.1101/413484

[73] Guo, Q., Lehmer, C., Martinez-Sanchez, A., Rudack, T., Beck, F., Hartmann, H., Perez-Berlanga, M., Frottin, F., Hipp, M.S., Hartl, F.U., Edbauer, D., Baumeister, W. and Fernandez-Busnadiego, R. (2018) In Situ Structure of Neuronal C9orf72 Poly-GA Aggregates Reveals Proteasome Recruitment. Cell, 172, 696-705, e612. https://doi.org/10.1016/j.cell.2017.12.030

[74] Dey, D., Siddiqui, S.I., Mamidi, P., Ghosh, S., Kumar, C.S., Chattopadhyay, S., Ghosh, S. and Banerjee, M. (2019) The Effect of Amantadine on an Ion Channel 
Protein from Chikungunya Virus. PLoS Neglected Tropical Diseases, 13, e0007548. https://doi.org/10.1371/journal.pntd.0007548

[75] Hrabe, T., Chen, Y., Pfeffer, S., Cuellar, L.K., Mangold, A.V. and Forster, F. (2012) PyTom: A Python-Based Toolbox for Localization of Macromolecules in Cryo-Electron Tomograms and Subtomogram Analysis. Journal of Structural Biology, 178, 177-188. https://doi.org/10.1016/j.jsb.2011.12.003

[76] Himes, B.A. and Zhang, P. (2018) emClarity: Software for High-Resolution CryoElectron Tomography and Subtomogram Averaging. Nature Methods, 15, 955-961. https://doi.org/10.1038/s41592-018-0167-Z

[77] Li, X., Radhakrishnan, A., Grushin, K., Kasula, R., Chaudhuri, A., Gomathinayagam, S., Krishnakumar, S.S., Liu, J. and Rothman, J.E. (2019) Symmetrical Organization of Proteins under Docked Synaptic Vesicles. FEBS Letters, 593, 144-153. https://doi.org/10.1002/1873-3468.13316

[78] Quade, B., Camacho, M., Zhao, X., Orlando, M., Trimbuch, T., Xu, J., Li, W., Nicastro, D., Rosenmund, C. and Rizo, J. (2019) Membrane Bridging by Munc13-1 Is Crucial for Neurotransmitter Release. Elife, 8, e42806. https://doi.org/10.7554/eLife.42806

[79] Liu, X., Seven, A.B., Camacho, M., Esser, V., Xu, J., Trimbuch, T., Quade, B., Su, L., Ma, C., Rosenmund, C. and Rizo, J. (2016) Functional Synergy between the Munc13 C-Terminal C1 and C2 Domains. Elife, 5, e13696. https://doi.org/10.7554/eLife.13696

[80] Ogawa, Y., Oses-Prieto, J., Kim, M.Y., Horresh, I., Peles, E., Burlingame, A.L., Trimmer, J.S., Meijer, D. and Rasband, M.N. (2010) ADAM22, a Kv1 Channel-Interacting Protein, Recruits Membrane-Associated Guanylate Kinases to Juxtaparanodes of Myelinated Axons. Journal of Neuroscience, 30, 1038-1048. https://doi.org/10.1523/JNEUROSCI.4661-09.2010

[81] Charles, P., Tait, S., Faivre-Sarrailh, C., Barbin, G., Gunn-Moore, F., Denisenko-Nehrbass, N., Guennoc, A.-M., Girault, J.-A., Brophy, P.J. and Lubetzki, C. (2002) Neurofascin Is a Glial Receptor for the Paranodin/Caspr-Contactin Axonal Complex at the Axoglial Junction. Current Biology, 12, 217-220. https://doi.org/10.1016/S0960-9822(01)00680-7

[82] Davis, J.Q., Lambert, S. and Bennett, V. (1996) Molecular Composition of the Node of Ranvier: Identification of Ankyrin-Binding Cell Adhesion Molecules Neurofascin (Mucin+/Third FNIII Domain-) and NrCAM at Nodal Axon Segments. Journal of Cell Biology, 135, 1355-1367. https://doi.org/10.1083/jcb.135.5.1355

[83] Lau, C., Hunter, M.J., Stewart, A., Perozo, E. and Vandenberg, J.I. (2018) Never at Rest: Insights into the Conformational Dynamics of Ion Channels from Cryo-Electron Microscopy. The Journal of Physiology, 596, 1107-1119. https://doi.org/10.1113/JP274888

[84] Baraban, M., Koudelka, S. and Lyons, D.A. (2018) $\mathrm{Ca}^{2+}$ Activity Signatures of Myelin Sheath Formation and Growth in Vivo. Nature Neuroscience, 21, 19-23. https://doi.org/10.1038/s41593-017-0040-x

[85] Krasnow, A.M., Ford, M.C., Valdivia, L.E., Wilson, S.W. and Attwell, D. (2018) Regulation of Developing Myelin Sheath Elongation by Oligodendrocyte Calcium Transients in Vivo. Nature Neuroscience, 21, 24-28. https://doi.org/10.1038/s41593-017-0031-y

[86] Saab, A.S., Tzvetavona, I.D., Trevisiol, A., Baltan, S., Dibaj, P., Kusch, K., Mobius, W., Goetze, B., Jahn, H.M., Huang, W., Steffens, H., Schomburg, E.D., Perez-Samartin, A., Perez-Cerda, F., Bakhtiari, D., Matute, C., Lowel, S., Griesinger, 
C., Hirrlinger, J., Kirchhoff, F. and Nave, K.A. (2016) Oligodendroglial NMDA Receptors Regulate Glucose Import and Axonal Energy Metabolism. Neuron, 91, 119-132. https://doi.org/10.1016/j.neuron.2016.05.016

[87] Lundgaard, I., Luzhynskaya, A., Stockley, J.H., Wang, Z., Evans, K.A., Swire, M., Volbracht, K., Gautier, H.O., Franklin, R.J., Charles, F.-C., Attwell, D. and Karadottir, R.T. (2013) Neuregulin and BDNF Induce a Switch to NMDA Receptor-Dependent Myelination by Oligodendrocytes. PLoS Biology, 11, e1001743. https://doi.org/10.1371/journal.pbio.1001743

[88] Emery, B. and Lu, Q.R. (2015) Transcriptional and Epigenetic Regulation of Oligodendrocyte Development and Myelination in the Central Nervous System. Cold Spring Harbor Perspectives in Biology, 7, a020461.

https://doi.org/10.1101/cshperspect.a020461 